\title{
Topological design of lightweight additively manufactured mirrors for space
}

\author{
Carolyn Atkins, Charlotte Feldman, David Brooks, \\ Stephen Watson, William Cochrane, \\ Melanie Roulet, Emmanuel Hugot, Mat Beardsley, \\ Michael Harris, Christopher Spindloe, Simon G. Alcock, \\ Ioana-Theodora Nistea, Christian Morawe, François Perrin
}

\section{Published version information:}

Citation: C Atkins et al. "Topological design of lightweight additively manufactured mirrors for space." Proceedings of SPIE, vol. 10706 (2018): 107060I. Is in Advances in Optical and Mechanical Technologies for Telescopes and Instrumentation III, proceedings of SPIE Astronomical Telescopes + Instrumentation 2018, Austin, Texas, United States, 10-15 Jun 2018.

DOI: $\underline{10.1117 / 12.2313353}$

(C) 2018 Society of Photo Optical Instrumentation Engineers (SPIE). One print or electronic copy may be made for personal use only. Systematic reproduction and distribution, duplication of any material in this publication for a fee or for commercial purposes, or modification of the contents of the publication are prohibited.

This version is made available in accordance with publisher policies. Please cite only the published version using the reference above. This is the citation assigned by the publisher at the time of issuing the APV. Please check the publisher's website for any updates. 


\section{Topological design of lightweight additively manufactured mirrors for space}

\section{Carolyn Atkins, Charlotte Feldman, David Brooks, Stephen Watson, William Cochrane, et al.}

Carolyn Atkins, Charlotte Feldman, David Brooks, Stephen Watson, William Cochrane, Melanie Roulet, Emmanuel Hugot, Mat Beardsley, Michael Harris, Christopher Spindloe, Simon G. Alcock, loana-Theodora Nistea, Christian Morawe, François Perrin, "Topological design of lightweight additively manufactured mirrors for space," Proc. SPIE 10706, Advances in Optical and Mechanical Technologies for Telescopes and Instrumentation III, 107060I (10 July 2018); doi: 10.1117/12.2313353

Event: SPIE Astronomical Telescopes + Instrumentation, 2018, Austin, Texas, United States 


\title{
Topological design of lightweight additively manufactured mirrors for space
}

\author{
Carolyn Atkins ${ }^{* a}$, Charlotte Feldman ${ }^{b}$, David Brooks ${ }^{c}$, Stephen Watson ${ }^{a}$, William Cochrane ${ }^{a}$, \\ Melanie Roulet $^{a, d}$, Emmanuel Hugot ${ }^{d}$, Mat Beardsley ${ }^{e}$, Michael Harris ${ }^{e}$, Christopher Spindloe ${ }^{f}$ \\ , Simon G. Alcock ${ }^{g}$, Ioana-Theodora Nistea ${ }^{g}$, Christian Morawe $^{h}$, François Perrin ${ }^{h}$ \\ ${ }^{a}$ UK Astronomy Technology Centre, Royal Observatory, Edinburgh, EH9 3HJ, UK \\ ${ }^{b}$ University of Leicester, Department of Physics and Astronomy, Leicester, LE1 7RH, UK \\ ${ }^{c}$ University College London, Department of Physics and Astronomy, London, WC1E 6BT, UK \\ ${ }^{d}$ Aix Marseille Univ, CNRS, CNES, LAM, Marseille, France \\ ${ }^{e}$ RAL Space, Harwell Science \& Innovation Campus, OX11 0QX, UK \\ ${ }^{f}$ Central Laser Facility, Harwell Science \& Innovation Campus, OX11 0QX, UK \\ ${ }^{g}$ Diamond Light Source, Harwell Science \& Innovation Campus, OX11 0QX, UK \\ ${ }^{h}$ European Synchrotron Radiation Facility, 71 Avenue des Martyrs, 38000 Grenoble, France
}

\begin{abstract}
Additive manufacturing (AM), more commonly known as 3D printing, is a commercially established technology for rapid prototyping and fabrication of bespoke intricate parts. To date, research quality mirror prototypes are being trialled using additive manufacturing, where a high quality reflective surface is created in a post-processing step. One advantage of additive manufacturing for mirror fabrication is the ease to lightweight the structure: the design is no longer confined by traditional machining (mill, drill and lathe) and optimised/innovative structures can be used. The end applications of lightweight AM mirrors are broad; the motivation behind this research is low mass mirrors for space-based astronomical or Earth Observation imaging. An example of a potential application could be within nano-satellites, where volume and mass limits are critical. The research presented in this paper highlights the early stage experimental development in AM mirrors and the future innovative designs which could be applied using AM.

The surface roughness on a diamond-turned $\mathrm{AM}$ aluminium $\left(\mathrm{AlSi}_{10} \mathrm{Mg}\right)$ mirror is presented which demonstrates the ability to achieve an average roughness of $\sim 3.6 \mathrm{~nm}$ root mean square (RMS) measured over a $3 \times 3$ grid. A Fourier transform of the roughness data is shown which deconvolves the roughness into contributions from the diamond-turning tooling and the AM build layers. In addition, two nickel phosphorus (NiP) coated $\mathrm{AlSi}_{10} \mathrm{Mg} \mathrm{AM}$ mirrors are compared in terms of surface form error; one mirror has a generic sandwich lightweight design at $44 \%$ the mass of a solid equivalent, prior to coating and the second mirror was lightweighted further using the finite element analysis tool topology optimisation. The surface form error indicates an improvement in peak-to-valley (PV) from $323 \mathrm{~nm}$ to $204 \mathrm{~nm}$ and in RMS from $83 \mathrm{~nm}$ to $31 \mathrm{~nm}$ for the generic and optimised lightweighting respectively while demonstrating a weight reduction between the samples of $18 \%$. The paper concludes with a discussion of the breadth of AM design that could be applied to mirror lightweighting in the future, in particular, topology optimisation, tessellating polyhedrons and Voronoi cells are presented.
\end{abstract}

Keywords: Additive manufacturing, 3D printing, mirror fabrication, surface roughness, lightweighting design, topology optimisation

\section{INTRODUCTION}

Additive manufacturing (AM; 3D printing) is a fabrication method that can build a part layer-upon-layer using a variety of materials (metal, ceramic, plastic) and methods (laser sintering, stereolithography, etc.) depending on the end use of the part. ${ }^{1}$ AM is particularly appealing at present as it can create structures that would not be

*Email: carolyn.atkins@stfc.ac.uk

Advances in Optical and Mechanical Technologies for Telescopes and Instrumentation III, edited by Ramón Navarro, Roland Geyl, Proc. of SPIE Vol. 10706, 107060I · @ 2018 SPIE CCC code: $0277-786 X / 18 / \$ 18 \cdot$ doi: $10.1117 / 12.2313353$ 
possible via traditional machining (mill, drill and lathe) and there is an appreciable reduction in waste material as only the primary structure and potentially some excess support structures are required. AM was initially the domain of rapid prototyping; however, increasingly it is being used to create bespoke parts for real-world applications, with the advantage of innovative design being one of the chief benefits. AM mirrors have been under investigation for several years with encouraging results obtained using aluminium and titanium AM alloys. ${ }^{2-4}$ The advantage of AM for mirror fabrication, and particularly towards space-based astronomical applications, is the ability to create innovative lightweighting designs which would not be possible via traditional methods.

Traditionally, there are three variants of lightweight mirror design ${ }^{5}$ as shown in Figure 1: 1) contoured back mirrors, 2) open back mirrors, and 3) sandwich mirrors. Each of these variants have advantages and disadvantages and a comprehensive comparison is provided by Schwertz et al. (2012). ${ }^{6}$ Contoured back mirrors are relatively easy to fabricate and to mount, but they do not offer the best weight reduction. Open back mirrors and sandwich mirrors offer a better weight reduction, but conversely are difficult and expensive to fabricate. The difference between open back mirrors, which are commonly used in lightweight mirror design, and sandwich mirrors, is that sandwich mirrors are stiffer and therefore offer minimal deflection per weight and height than open back mirrors, but they are more difficult to fabricate and mount, which in turn leads to increase cost.

a)

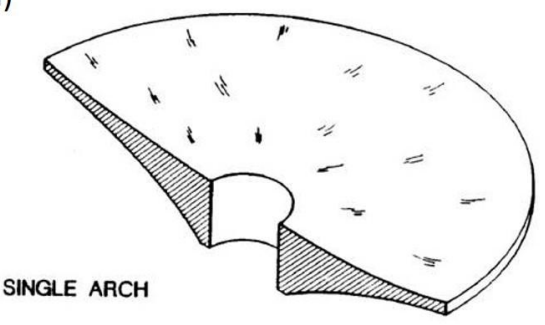

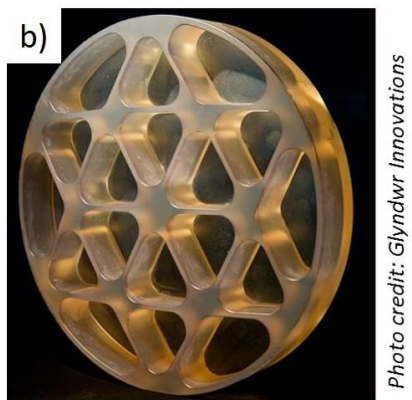

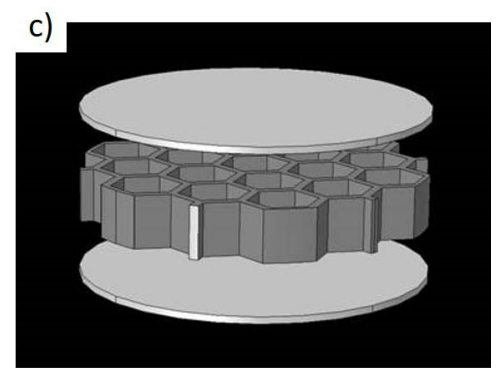

Figure 1. a) - example of a contoured back lightweight mirror design (image credit: Vukobratovich $\left.(1999)^{5}\right)$; $b$ ) - example of an open back mirror (image credit: Glyndŵw Innovations); and c) - an example of an sandwich mirror demonstrating the three individual components that are bonded together.

Therefore sandwich mirrors offer the optimum lightweighting performance out of the three traditional variants and if AM can be used to print the three individual components of the mirror in one part, the disadvantages of fabrication complexity and expense are reduced. Furthermore, the challenge in mounting sandwich mirrors can be counteracted via the inclusion of integrated mounting, as the only limitation on the mirror design beyond AM machine build volume, is in the ability to generate the mirror surface. Therefore, additive manufacturing offers a direct path to produce lightweight mirrors that are structurally more rigid and easier to fabricate for future astronomical applications.

This paper presents the conclusion and a continuation of a UK Space Agency (UKSA) funded project on 'Additive manufacturing for space-based optics' which was presented at SPIE Optics+Photonics in 2017.7,8 The objective of this research was to investigate different AM materials (plastics and metals) and different AM methods (laser sintering and stereolithography) towards lightweight mirrors for space. A series of small test samples were created, polished and their optical surfaces evaluated. In the final section of Atkins et al. $(2017)^{7}$ was the design of an optimised lightweighting design modelled using the finite element analysis (FEA) tool topology optimisation. This paper concludes this research with an analysis of the polished non-optimised and optimised samples (Section 3) in terms of surface form error and evaluating the polishing print-through effect. Following the conclusion of the UKSA project, an excess AM aluminium sample was diamond-turned to determine the mirror quality that could be achieved on a diamond-turned surface in comparison to a polished AM aluminium surface, particularly in regard to surface roughness - Section 2. Finally, Section 4 will discuss the breadth of lightweighting design that can be applied using AM towards lightweight mirrors. The organisation of this paper follows an increase in feature size: starting with the microscopic roughness and concluding with macroscopic structral design. 


\section{DIAMOND-TURNED MIRROR}

The sample for diamond-turning was $40 \mathrm{~mm}$ diameter, $6 \mathrm{~mm}$ in height and had $4 \mathrm{~mm}$ of inner lightweighting in the form of arches as shown in Figure 2. The design requirements for the sample are described in Atkins et al. (2017), ${ }^{7}$ but the optical prescription was a flat for ease of measurement. The sample was printed commercially using metal laser sintering (MLS) in the aluminium alloy $\mathrm{AlSi}_{10} \mathrm{Mg}$ and was lightweighted to $~ 44 \%$ of a solid equivalent (9g arches lightweighting, 20.4g solid). The RAL Space Precision Development Facility (PDF) performed the diamond-turning.

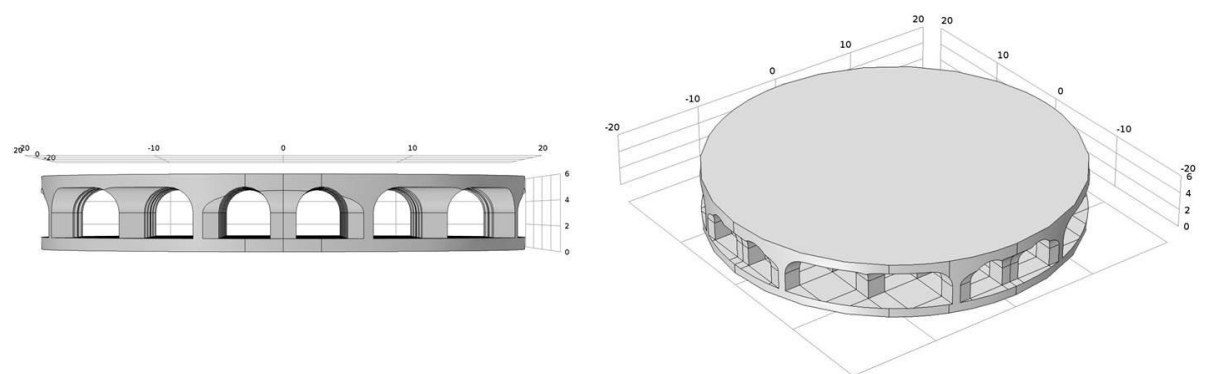

Figure 2. A computer aided design of the initial sample: $40 \mathrm{~mm}$ diameter, $6 \mathrm{~mm}$ in height

The primary objective in the diamond-turning study was to investigate the quality of the reflective surface that could be generated using the PDF single point diamond turning (SPDT) machine, there were no expectations in the resultant surface form error or surface roughness. The SPDT was used to generate a flat optical surface on the AM substrate. Prior to diamond-turning the sample had been bead blasted to remove the majority of the post-print roughness. A series of Taylor Hobson Form Talysurf Intra profiles were measured over a $38 \mathrm{~mm}$ length and intersecting the centre of the part at $0^{\circ}, 45^{\circ}$ and $90^{\circ}$ to evaluate the pre diamond-turning surface quality. To extract the surface roughness data from the form error (defined as the deviation from the optical prescription), the low frequency component of the data was removed using a $2.5 \mathrm{~mm}$ Fourier filter cut-off; the resultant peak-to-valley (PV) and root mean square (RMS) were $17.99 \mu \mathrm{m}$ and $1.74 \mu \mathrm{m}$ respectively averaged over the three orientations; a more comprehensive discussion of the data analysis is provided within Roulet et al. (2018). ${ }^{9}$ In regard to form error, qualitatively the part was relatively flat post bead-blast with no dominant periods or curvature. Figure $3 a$ ) highlights the aluminium sample prior to diamond-turning.
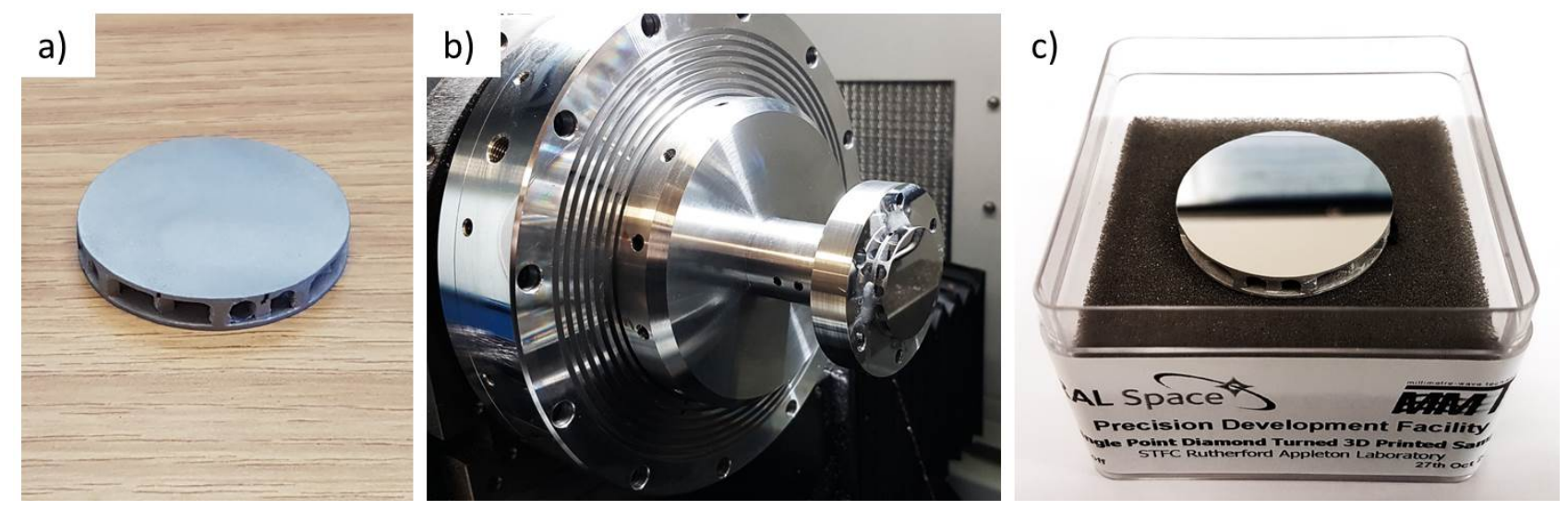

Figure 3. (a) - the bead-blasted AM aluminium sample prior to diamond-turning, (b) - the sample in situ upon the SPDT machine and (c) - the finished diamond-turned sample.

A $0.504 \mathrm{~mm}$ radius tool tip was used at a pitch of $0.006 \mathrm{~mm}$ by the SPDT machine to generate the reflective surface. Only a single pass with the SPDT was performed due to time and resource limitations. 


\subsection{Metrology: surface form error}

The form error of the mirror surface was measured at the UK Astronomy Technology Centre using a Zygo interferometer at $\lambda=633 \mathrm{~nm}$ and because only a single pass with the SPDT was possible, the form error does not represent the optimum surface that could be achieved. The raw surface is shown in Figure $4 a$ ) and $b$ ), the surface is concave with a PV of $\sim 600 \mathrm{~nm}$. The centre and the edge indicate loss of data due to SPDT marks in those regions. Figure $4 c$ ) is a representation of the simulated surface form error by fitting Zernike terms up to $Z_{8}$ (spherical defocus). Figure $4 d$ ) highlights the residual surface form error after Zernike subtraction with a RMS of $\sim 22 \mathrm{~nm}$, the arches lightweighting structure is apparent.

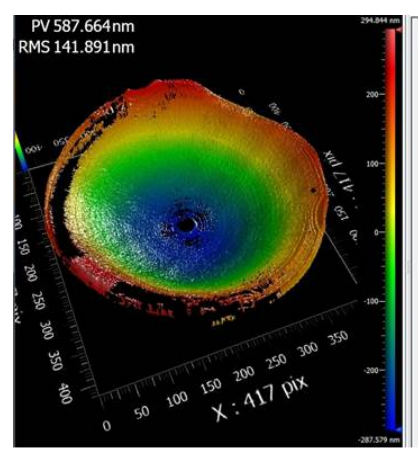

a)

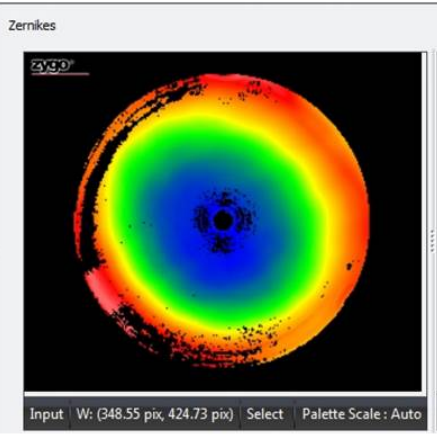

b)

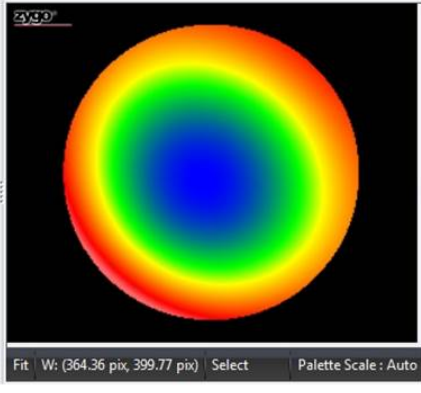

c)

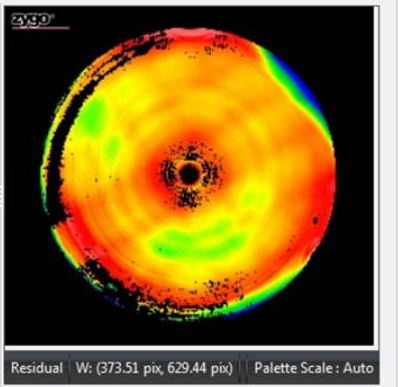

d)

Figure 4. $a$ and $b$ - raw measurement data, $(c)$ - Zernike fit: tilt, defocus, astigmatism, coma and spherical defocus, $(d)$ residuals after Zernike removal.

\subsection{Metrology: surface roughness}

The surface roughness of the mirror was measured at the Optical Metrology Laboratory at the Diamond Light Source Ltd. using a Bruker Contour GT-X stitching microinterferometer. Located on the mirror centre, a $3 \times$ 3 grid with $10 \mathrm{~mm}$ spacing was used to sample the mirror. The grid was evaluated at three magnifications: $5 \times$, $10 \times$ and $50 \times$. The images of each magnification are show in Figures 5, 6 and 7 respectively.

In each of the three figures the spiral form of the SPDT tooling is evident. In addition, lines are observed in-plane with the horizontal axis in all the $3 \times 3$ images for each magnification. The average spacing between the lines is $\sim 50 \mu \mathrm{m}$ and with a range between $\min \sim 10 \mu \mathrm{m}$ and $\max \sim 100 \mu \mathrm{m}$. It is assumed that these lines represent the build layers used during AM manufacturing; the AM manufacturer for this part quoted $\sim 50 \mu m$ as the build layer. It is also known that this part was built in the vertical orientation, which would account for the near $50 \mu \mathrm{m}$ layer spacing. The cause of the variability in thickness of the layers is unknown.

A Fourier analysis of the data (Figure 8) highlighted a dominant frequency at $0.006 \mathrm{~mm}$ which correlates to the lay-lines induced by SPDT. Figure 8(a) and Figure 8(b) depict the build lines and the SPDT lay lines in parallel and perpendicular to one another respectively; the comparison of the two plots deconvolves the two roughness components into the low spatial frequency build roughness and the high spatial frequency SPDT roughness.

The numerical evaluation of roughness for the three magnifications is shown in Table 1. The average Sq (RMS) value for the three magnifications excluding the central points is $3.6 \mathrm{~nm}$. In comparison to previous AM aluminium parts, which range from a Sq of $6 \mathrm{~nm}-22 \mathrm{~nm},{ }^{2,3,7}$ the result demonstrates the potential AM aluminium mirrors for visible and infrared (IR) applications. Quantifying in terms of total integrated scatter ${ }^{10}$ (TIS) for the mirror and defined by the equation TIS $=1-\exp \left(-(4 \pi \sin \theta \sigma / \lambda)^{2}\right)$, where $\theta=$ angle of incidence to the surface $\left(90^{\circ}\right), \sigma=R M S$ roughness and $\lambda=$ wavelength; for $550 \mathrm{~nm}$ (visible spectrum) at the measured 3.6nm RMS the TIS is $\sim 0.7 \%$ and similarly, for $2500 \mathrm{~nm}$ (thermal IR), the TIS equals $\sim 0.03 \%$. Assuming that the mid- and low-spatial frequencies can be controlled within the required specifications, AM aluminium mirrors do have the potential for visible and IR applications in the future despite the presence of the AM build layers. 

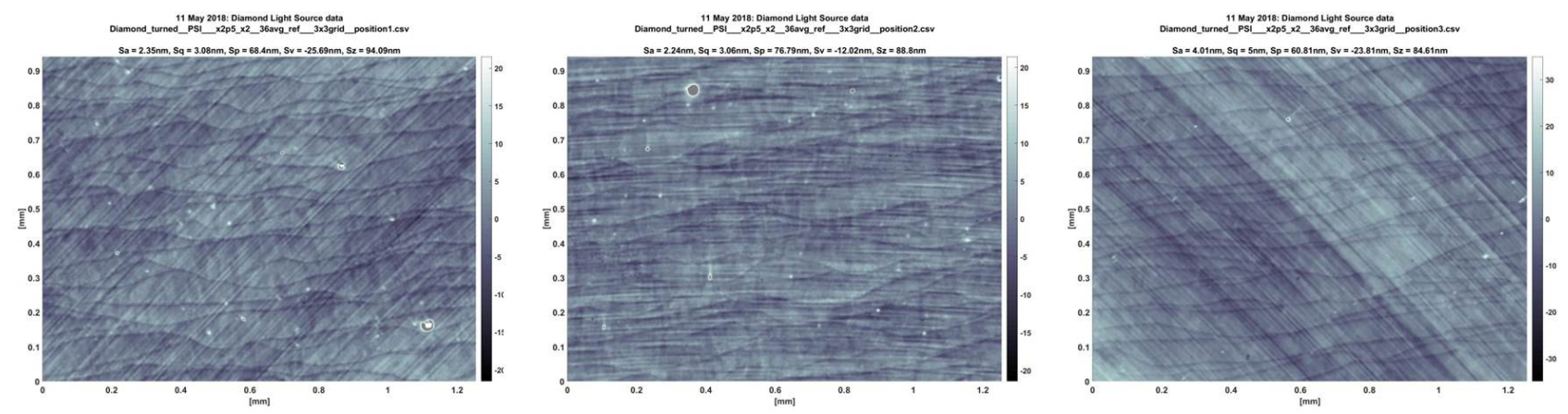

\section{5x magnification}
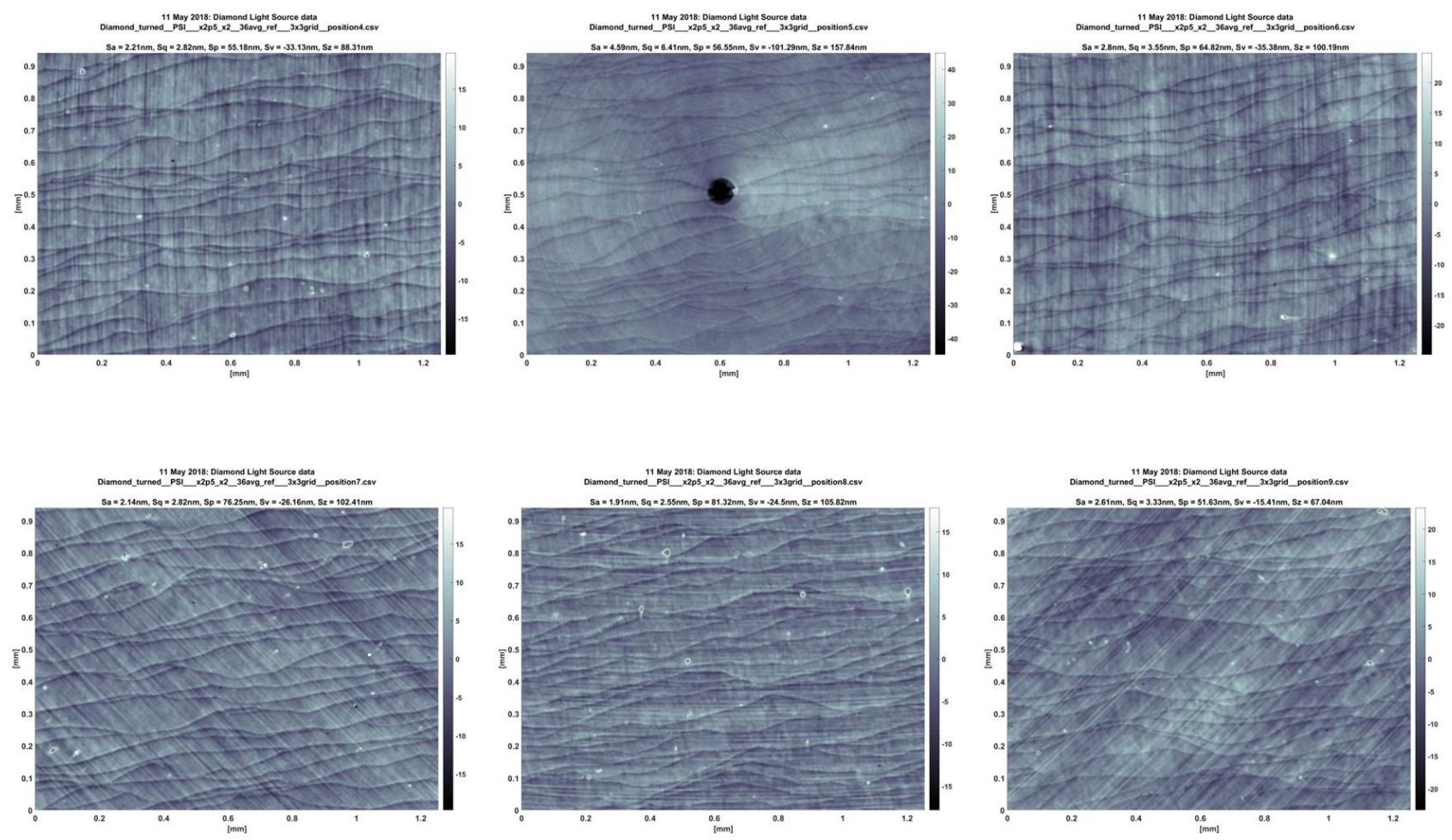

Figure 5. Bruker Contour GT-X stitching microinterferometer $3 \times 3$ grid at $5 \times$ magnification

Table 1. Average surface roughness values for the AM aluminium diamond-turned mirrors taken using the Bruker Contour GT-X stitching microinterferometer

\begin{tabular}{|l|l|l|l|l|l|l|l|l|l|}
\hline \multicolumn{9}{|c|}{ Surface roughness measurements } \\
\hline \multirow{2}{*}{ 3X3 Grid } & \multicolumn{3}{|c|}{ 5X magnification } & \multicolumn{3}{c|}{ 10X magnification } & \multicolumn{3}{c|}{$50 X$ magnification } \\
\cline { 2 - 10 } & Sa $[\mathrm{nm}]$ & Sq $[\mathrm{nm}]$ & Sz $[\mathrm{nm}]$ & Sa $[\mathrm{nm}]$ & Sq $[\mathrm{nm}]$ & Sz $[\mathrm{nm}]$ & Sa $[\mathrm{nm}]$ & Sq $[\mathrm{nm}]$ & Sz $[\mathrm{nm}]$ \\
\hline average & 2.53 & 3.28 & 91.41 & 2.83 & 3.66 & 117.95 & 3.08 & 3.84 & 40.56 \\
\hline $\max$ & 4.01 & 5.00 & 105.82 & 3.85 & 4.80 & 232.40 & 3.92 & 4.92 & 66.25 \\
\hline $\min$ & 1.91 & 2.55 & 67.04 & 2.45 & 3.14 & 60.27 & 2.47 & 3.10 & 24.08 \\
\hline
\end{tabular}

$\mathrm{Sa}=$ absolute mean, $\mathrm{Sq}=$ root mean square, $\mathrm{Sz}=$ peak-to-valley 

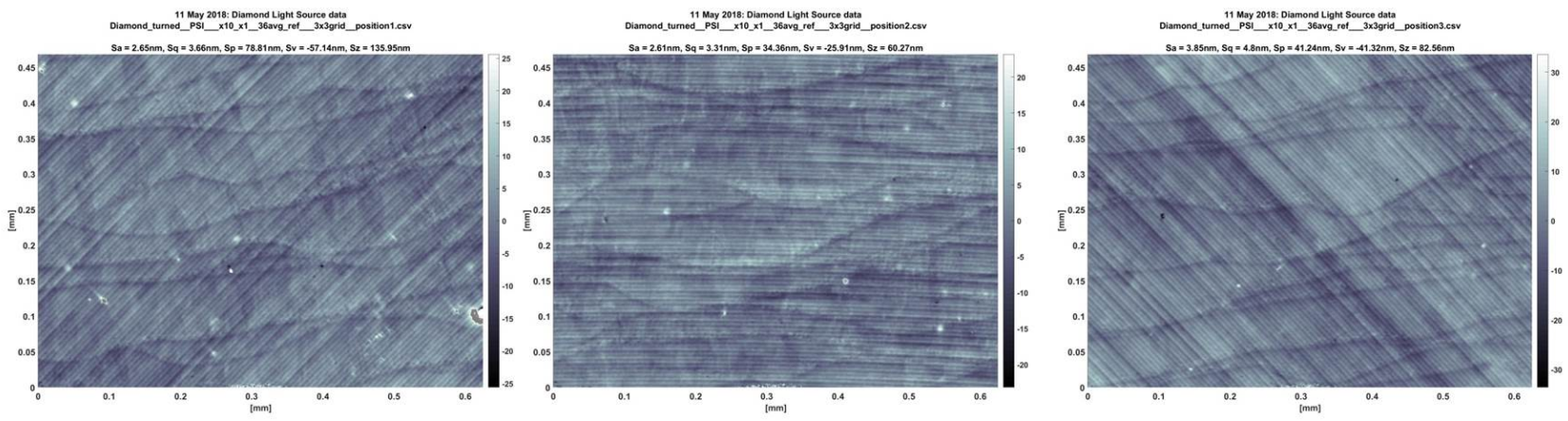

\section{0x magnification}
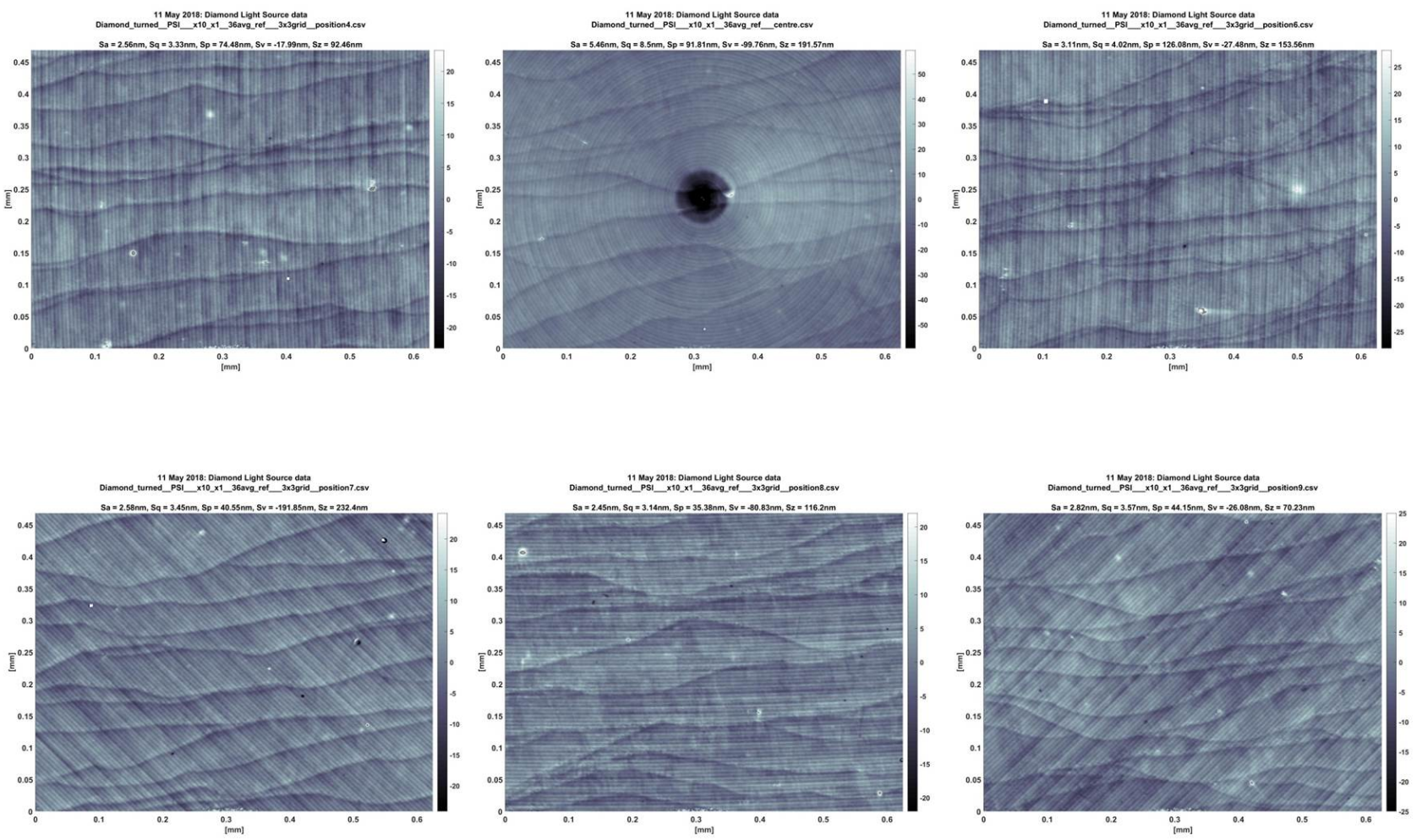

Figure 6. Bruker Contour GT-X stitching microinterferometer $3 \times 3$ grid at $10 \times$ magnification 

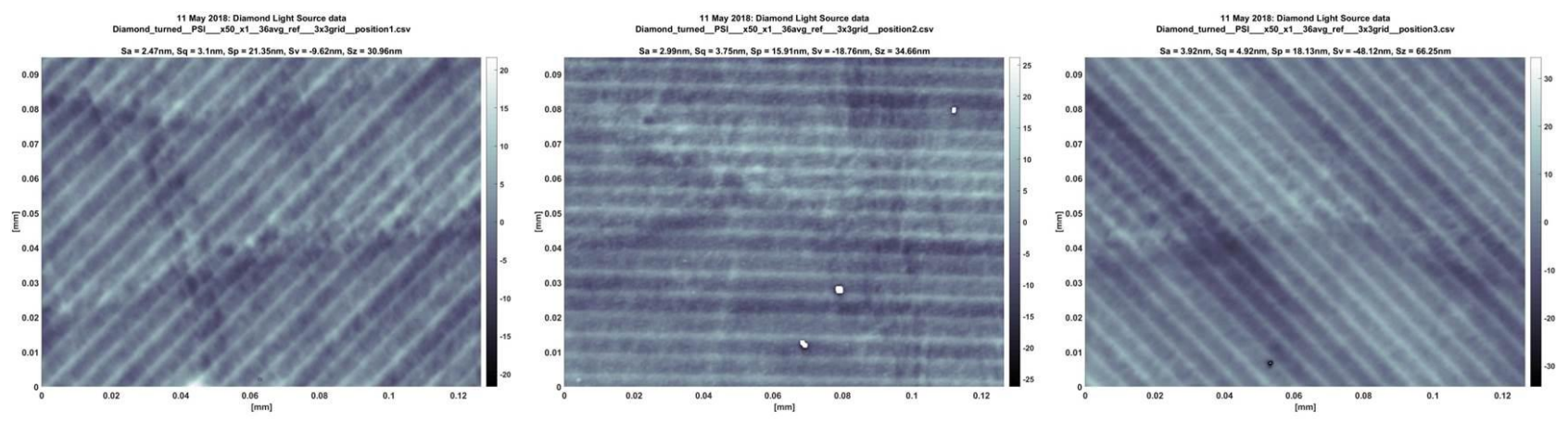

\section{0x magnification}
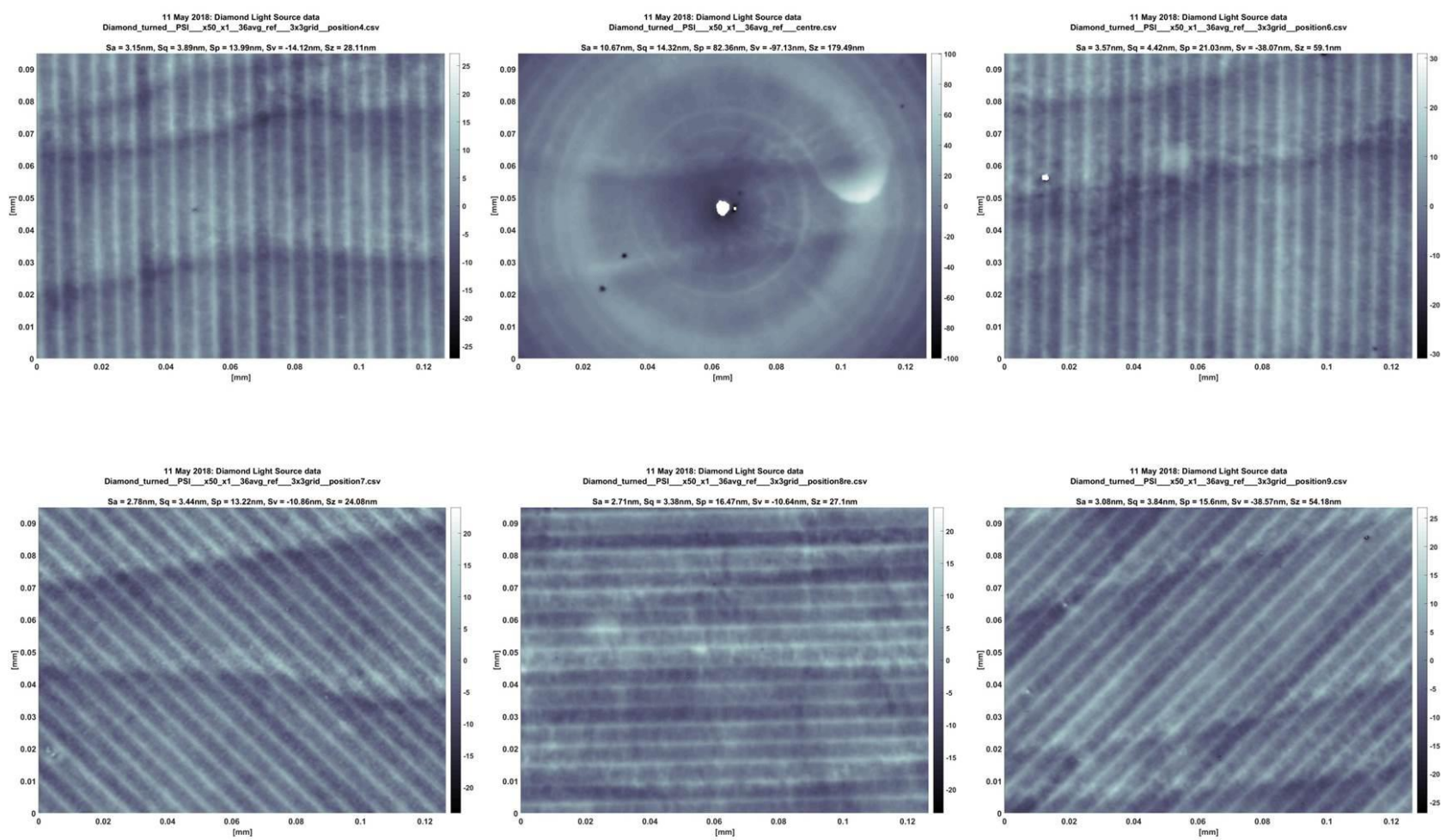

Figure 7. Bruker Contour GT-X stitching microinterferometer $3 \times 3$ grid at $50 \times$ magnification 
11 May 2018: Diamond Light Source data Diamond_turned_PSI__x10_x1_36avg_ref__3 3 3grid_position $8 . c s v$
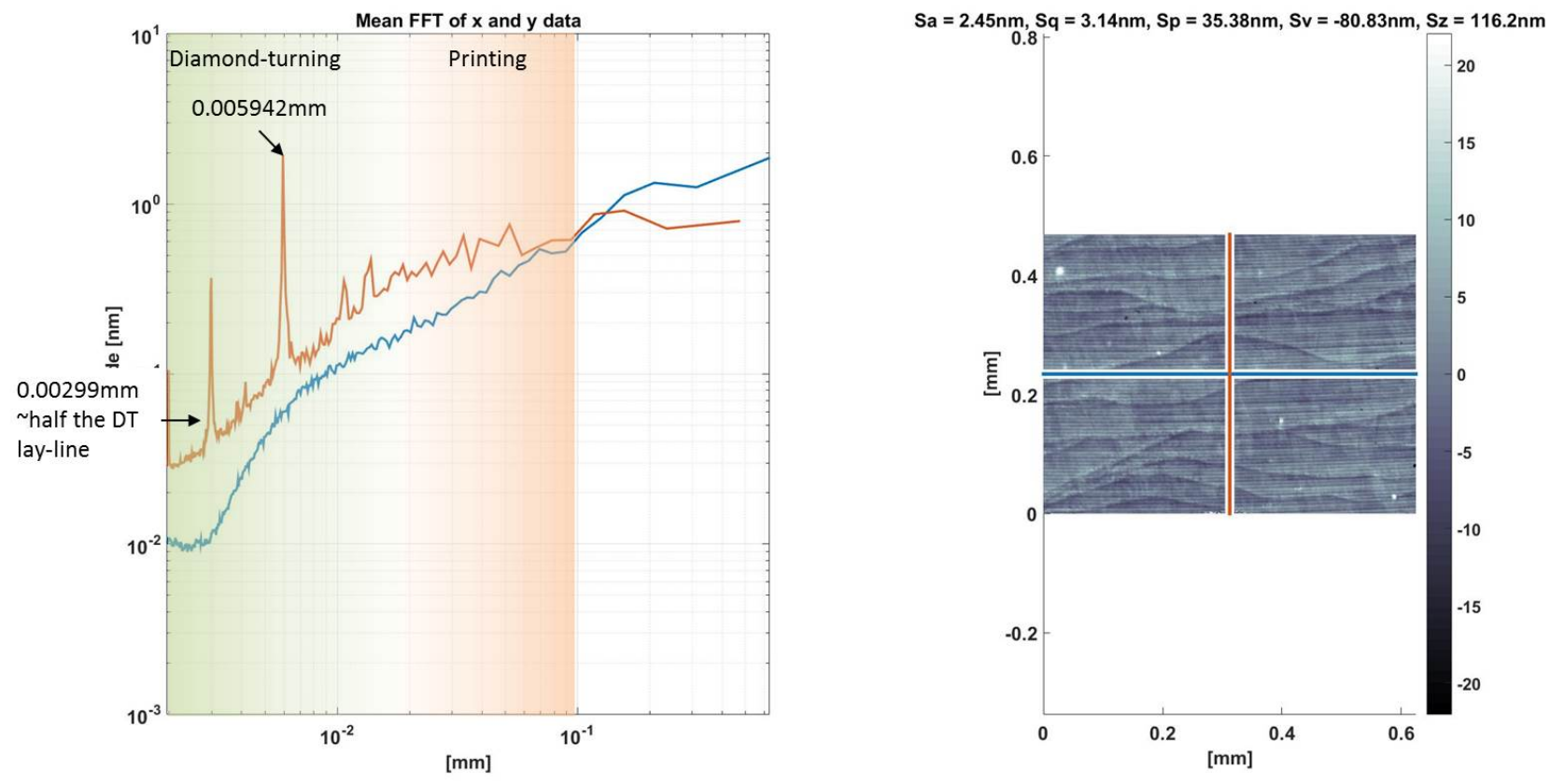

(a) Lay-lines approximately parallel to the AM build lines
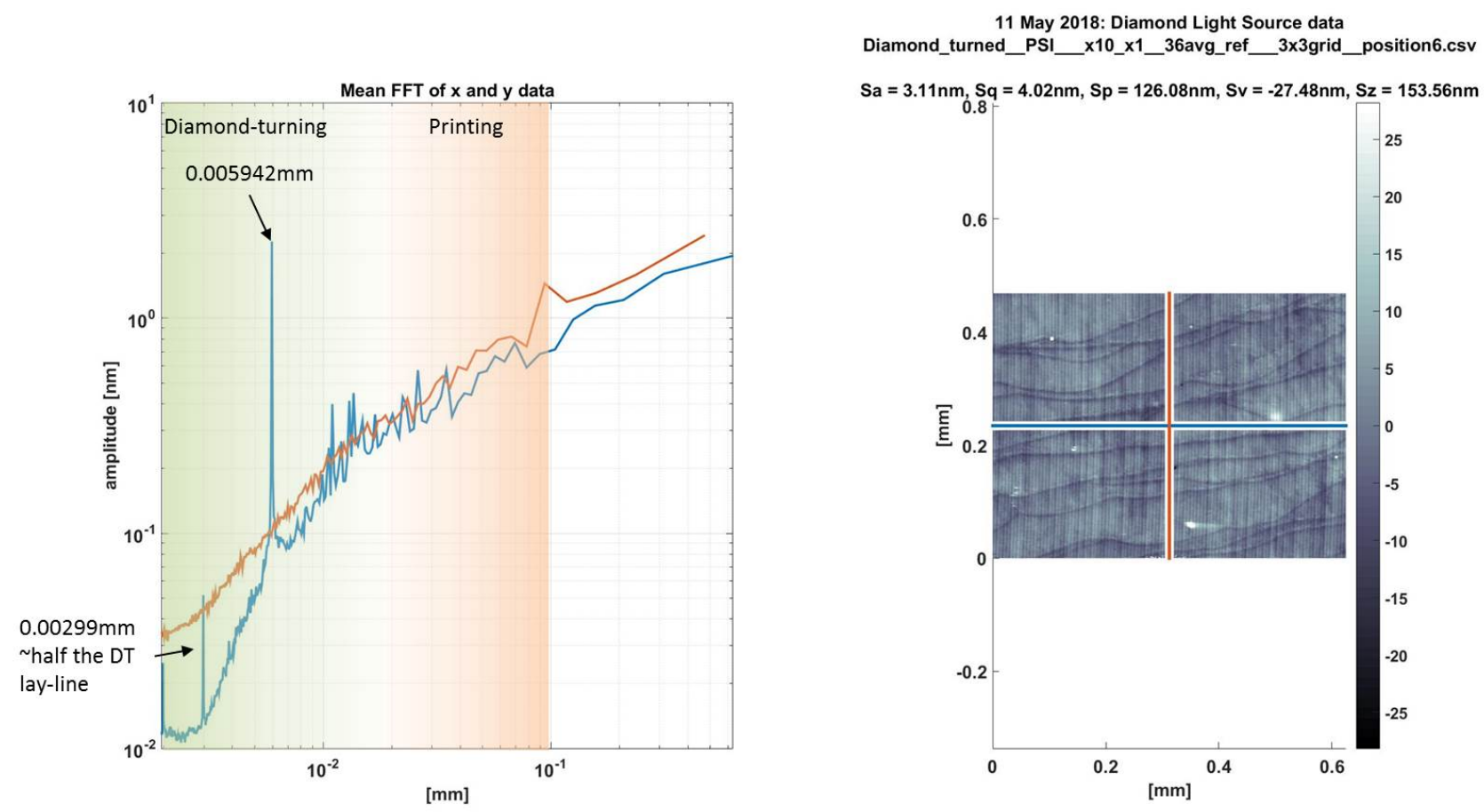

(b) Lay-lines approximately perpendicular to the AM build lines

Figure 8. Figures $8(\mathrm{a})$ and $8(\mathrm{~b})$ highlight the average Fourier transform of the $10 \times$ magnification data in where the SPDT lay-lines are parallel and perpendicular to the AM build lines respectively. 


\subsection{Conclusion}

The surface roughness data presented in this section demonstrates that an AM substrate can be directly machined to create a mirror surface for visible and infrared wavelengths. The build layers are visible in the mirror surface, but it is possible to mitigate these build effects through orientating the part differently within the AM build chamber. If the part was orientated in the horizontal direction, potentially the surface of the mirror could lie within one of these build layers - further investigation into the effect of build orientation and roughness is required. Diamond turning has demonstrated itself to be effective in generating low surface roughness mirror surfaces, future work will focus on using iterations of metrology and diamond-turning to minimise the surface form error of subsequent mirrors.

\section{LIGHTWEIGHTING COMPARISON: NON-OPTIMISED VERSUS OPTIMISED}

The previous section considered the roughness on the AM substrate, whereas the focus in this section is shifted towards mass reduction and evaluation of surface form error. Two samples are compared: a non-optimised generic lightweighting structure and an optimised lightweighting structure. Interferometric and profilometry data is presented.

\subsection{Non-optimised sample}

The design of the non-optimised sample was the same as the design of the arches sample in Figure 2. The part was $40 \mathrm{~mm}$ in diameter, $6 \mathrm{~mm}$ in height with $4 \mathrm{~mm}$ of lightweighting sandwiched between two solid face plates. The part was printed in $\mathrm{AlSi}_{10} \mathrm{Mg}$ via MLS and coated in NiP. The NiP coating allowed the emphasis to focus on the form error achieved on the AM part, as opposed to the surface roughness since NiP is commonly used to generate high quality mirror components.

\subsection{Optimised sample}

The goal with the optimised sample was to reduce the weight of the part relative to the non-optimised sample while maintaining the same aperture area and mirror quality. The face plates of the sandwich mirror were reduced in thickness to $0.5 \mathrm{~mm}$ and the lightweighting was optimised to mitigate the subsequent loss in stiffness in the face plates. The finite element analysis (FEA) tool topology optimisation (TO) was used to design the new lightweighting by minimising the total internal strain energy of the part for an applied polishing pressure, mass reduction and physical constraints. A thorough description of how TO was used to iterate to an optimised design has been previously described $;^{7}$ however, a brief overview of the procedure is presented in Figure 9.

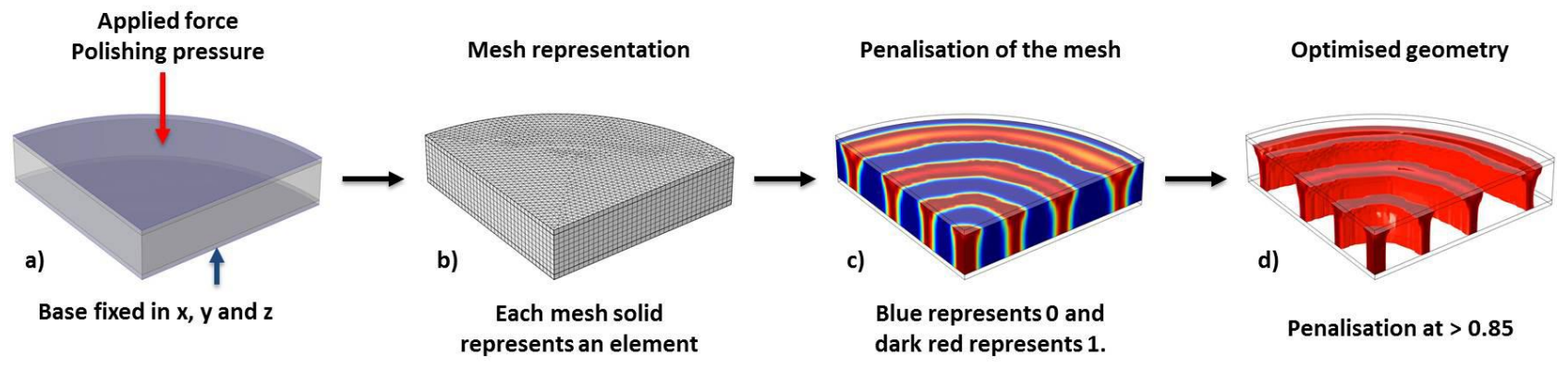

Figure 9. An example in the use of topology optimisation in the design of the sample lightweighting to minimise polishing quilting effect. a) the boundary conditions and the optimisation constraints (i.e. mass removal) are defined within the FEA model; b) the part is meshed - a triangular prism mesh is used in this example; c) the optimisation penalises the mesh elements to either 1 (solid) or 0 (void) during the iterations, the optimsation is complete when the solution converges; and d) the void elements are removed from the solution, which results in the optimised geometry.

Figure 10a) and b) highlight the design of the optimised sample; the lightweighting is a series of co-axial tapered rings radiating from a central pillar. Figure 10c) depicts the machined base plate of the sample. The optimised sample was printed inAlSi ${ }_{10} \mathrm{Mg}$ and the two face plates milled to $0.5 \mathrm{~mm}$, the sample was then coated in NiP prior to polishing, as shown in Figure 10d). 

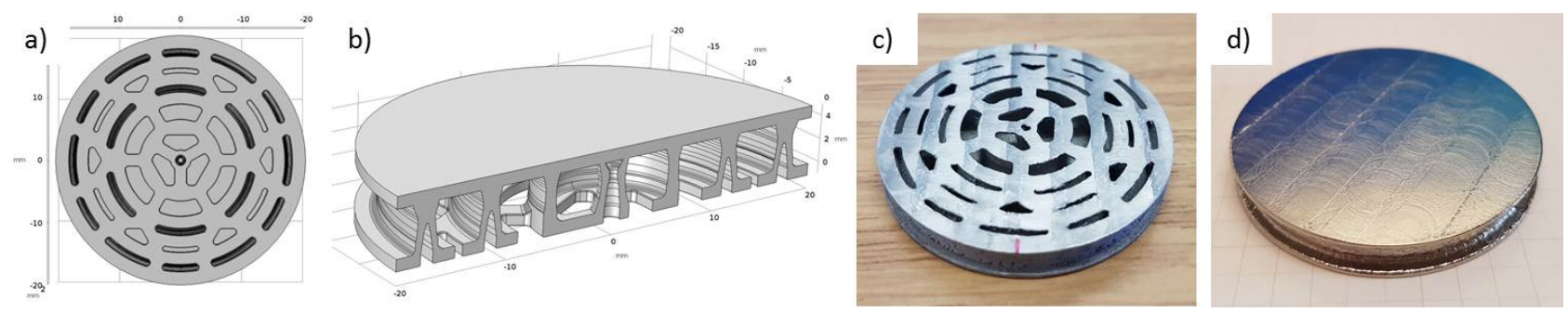

Figure 10. a) the lightweighted base plate design to allow unsolidified material removal; b) the core lightweighting design which was adapted from the solution in Figure 9; c) the AM aluminium printed part - base plate showing; and d) the $\mathrm{NiP}$ coated part - top plate showing.

\subsection{Polished samples}

Both the non-optimised and the optimised samples were ground and polished by hand at the University College London Optical Science Laboratory (OSL). The finished mirrors are shown in Figure 11. The weight comparison between the two finished mirrors is $14.3 \mathrm{~g}$ and $11.7 \mathrm{~g}$ for the non-optimised and the optimised designs respectively. However, some of the weight reduction gained in the optimised design has been lost due to the increased surface area which leads to an increase in the amount of the NiP coating deposited.
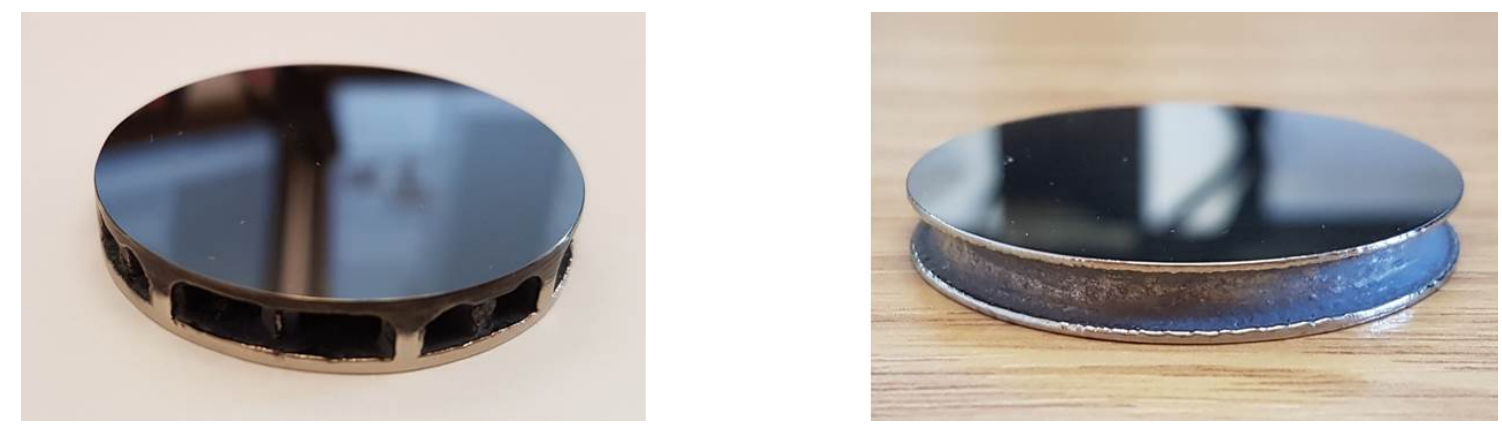

Figure 11. A comparison between the polished non-optimised (left) and optimised (right) mirror samples.

\subsection{Metrology: interferometry}

Interferometry data was taken at OSL during the polishing phase as a means to iterate towards a converge polishing result. The interferograms presented in Figure 12 are independent metrology data taken at the European Synchrotron Radiation Facility (ESRF) metrology laboratory on a ZYGO GPI - XPZ $(\lambda=632.8 \mathrm{~nm})$ interferometer. Qualitatively the non-optimised design has a low order oval concave form, whereas the optimised design is flatter with an obvious low-mid spatial frequency present. The PV and RMS of the non-optimised mirror is $323 \mathrm{~nm}$ and $83 \mathrm{~nm}$ respectively in comparsion to $204 \mathrm{~nm}$ and $31 \mathrm{~nm}$ for the optimised mirror. The cause of the oval form in the non-optimised mirror is unknown, polishing iterations at OSL failed to remove this error. The imprinted ring structure of the optimised design is consistent with the underlying ring structure of the lightweigting in terms of form; however, the period of the ring structure in the interferogram does not match that of the lightweighting. In addition, the optimised sample does highlight what are suspected to be milling marks in the vertical axis of the interferogram; these were visible prior to polishing as shown in Figure 10d).

\subsection{Metrology: profilometry}

To complement the interferometry data, profilometry data was taken on a Taylor Hobson Form Talysurf Intra at Glyndŵr Innovations (Figure 13a)). Profilometry data was taken along four orientations $\left(0^{\circ}, 45^{\circ}, 90^{\circ}\right.$ and $135^{\circ}$ ) on both the non-optimised and the optimised parts as shown in Figure 13b). 

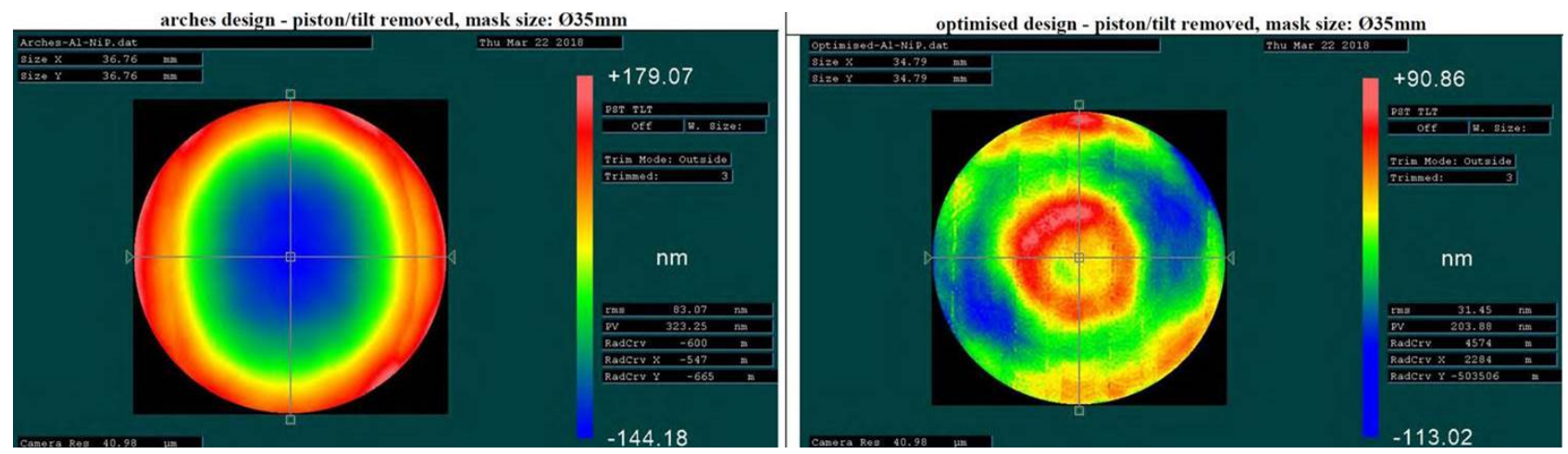

Figure 12. An interferometric comparison between the non-optimised (left) and the optimised (right) mirror samples.

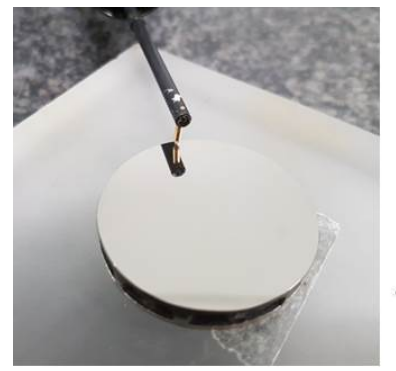

a)

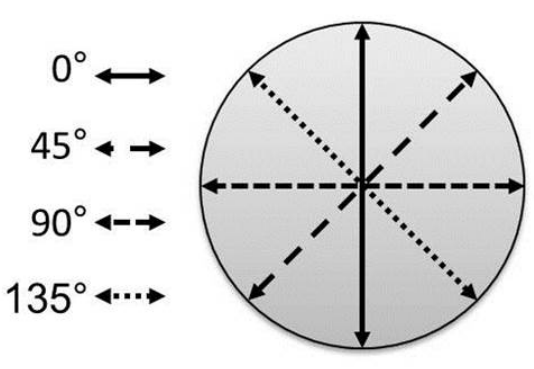

b)

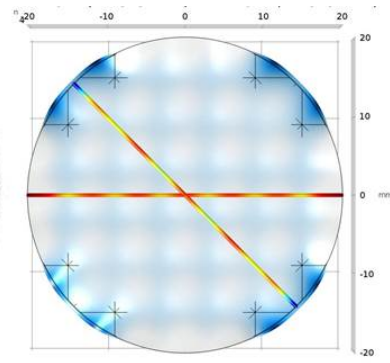

c)

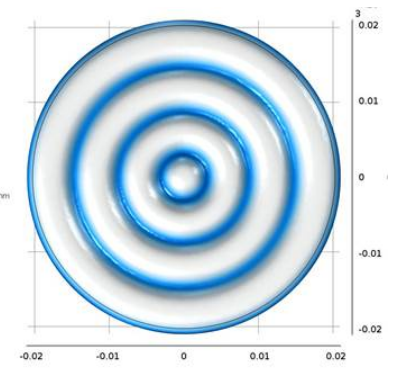

d)

Figure 13. a) - a sample being measured on the Talysurf Intra; b) - the measurement orientations for the profilometric measurements; c) - an FEA simulation of the expected polishing print-through map for a given polishing pressure using the non-optimised lightweighting, as observed the lightweighting period is different dependent upon measurement orientations; and $d$ ) - an FEA simulation of the expected polishing print-through map for a given polishing pressure using the optimised lightweighting, the co-axial ring strcuture lightweighting produces a period that is not dependent upon measurement orientation.

A Fourier analysis of the two data sets is shown in Figures 14 and 15. In regard to the non-optimised design, the polishing print-through effect (quilting) is observed in the measured data at the expected frequency shift for the measurement direction - measurements diagonal to the arch cells imprints a broader quilting effect upon the surface than measurements in-line with the arches. Despite the fact that there is a clear magnitude shift between the measured and FEA profiles, approximately $\sim 100 \times$, the alignment of the polishing print-through effects is encouraging, particularly as a means to mitigate the print-through for future lightweighting designs. The dominance of the oval effect is demonstrated by the profile maximum, in terms of magnitude, at the low order spatial frequency domain. In addition to the observed structural features, there is a mid-high spatial frequency peak shown at a wavelength of $\sim 1.3 \mathrm{~mm}$ which is suspected to be a polishing artefact.

The Fourier plot of the optimised lightweighting, shown in Figure 15, demonstrates the alignment of the measured print-through effect and that of the predicted FEA print-through; however, like the non-optimised design, the magnitude of the measured print-through is $\sim 100 \times$ the FEA value. In addition to the direct printthrough effect of the lightweighting, the dominant frequency seen in the interferometer image is also observed in the FEA data albeit at a lower magnitude. The answer as to why this frequency was the dominant one of the mirror surface is not yet thoroughly understood; however, like the non-optimised mirror, the agreement in the alignment of the observed features is encouraging in the design of future lightweighting.

\subsection{Conclusion}

A summary of the measured, or calculated, characteristics of the two lightweighted mirrors is provided in Table 2.

The presented physical characteristics highlight the weight reduction of the pre-coated optimised part, which is 

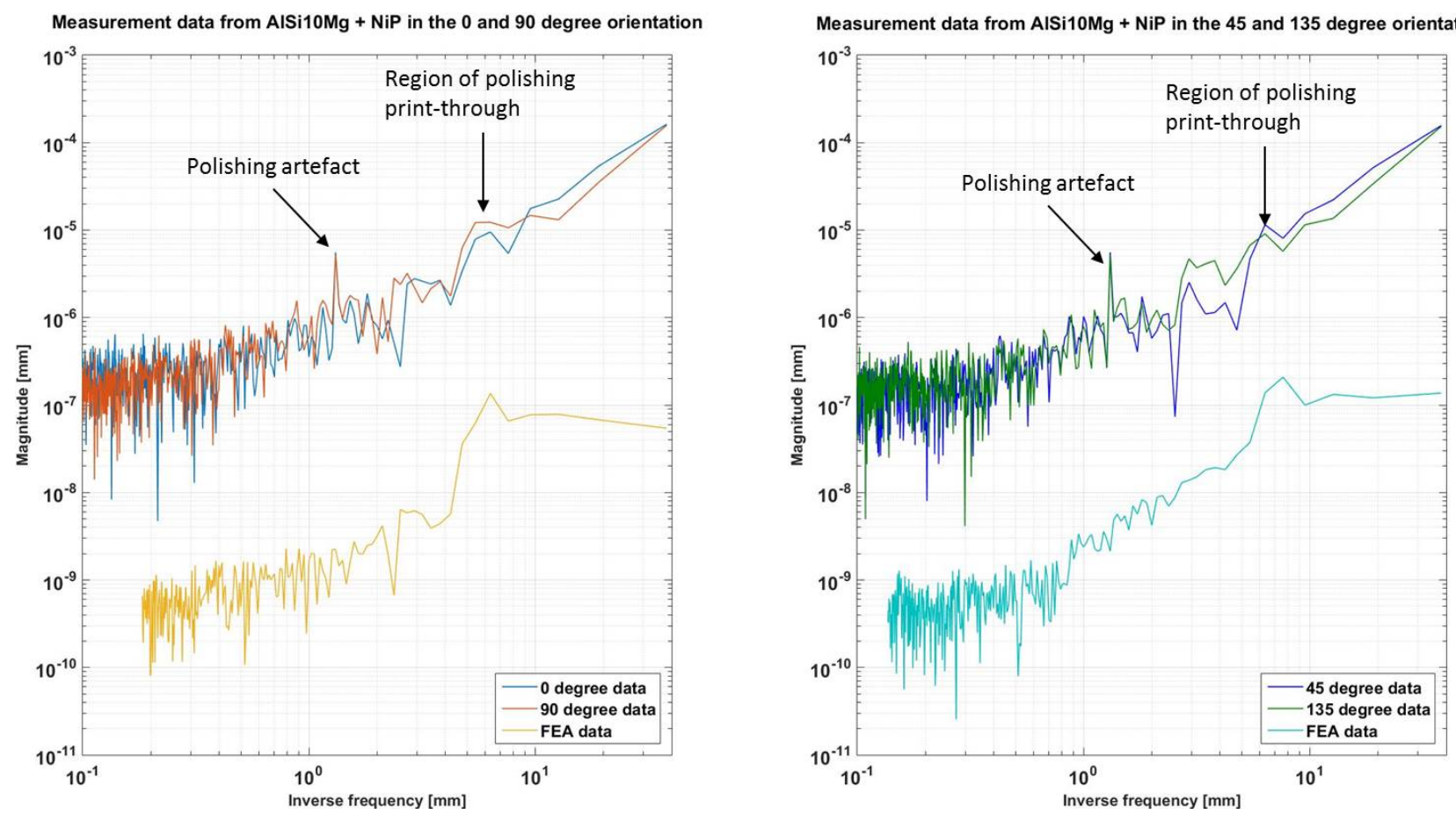

Figure 14. The measured FFT profiles in the $0^{\circ}$ and $90^{\circ}$ orientations (left) and the $45^{\circ}$ and $135^{\circ}$ orientations (right). In both cases the measured profiles have been compared against the respective FEA profiles highlighted in Figure 13c).
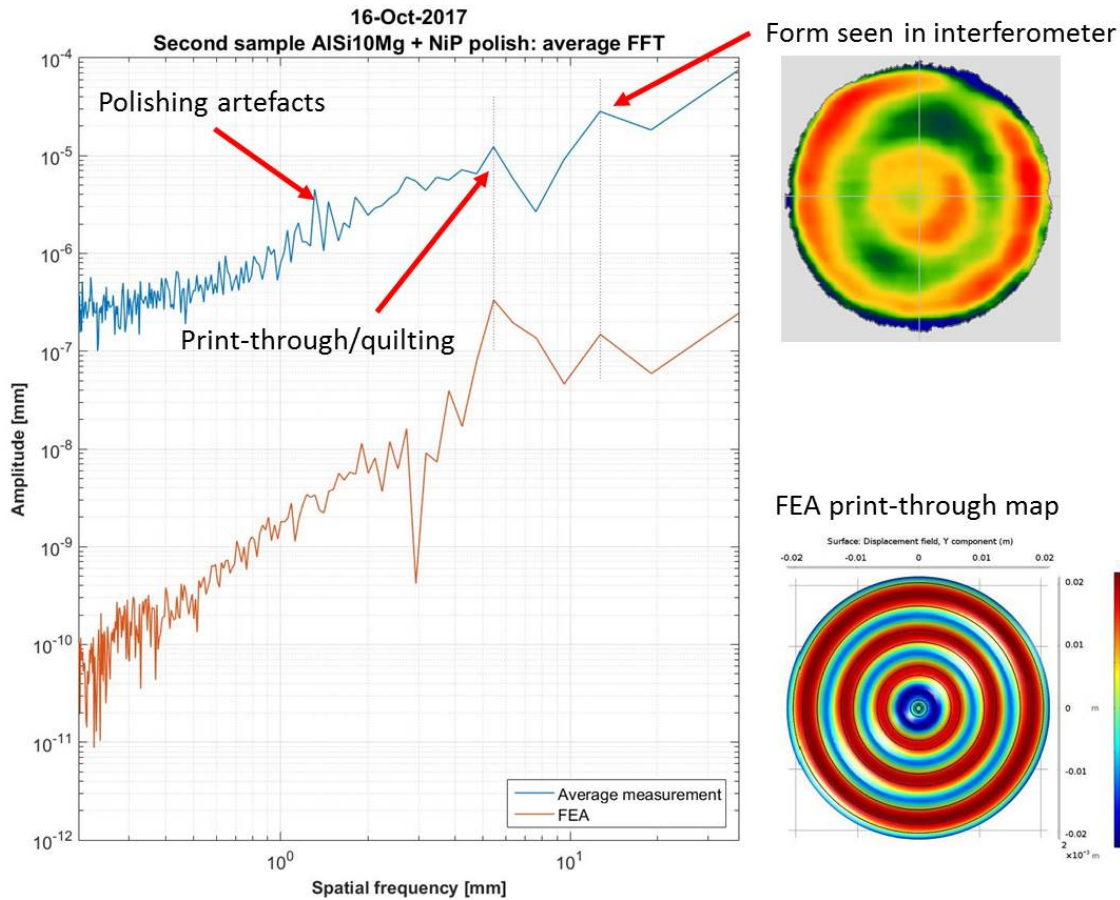

FEA print-through map

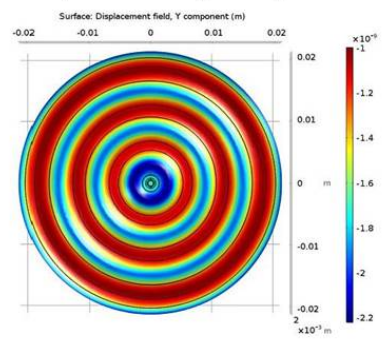

Figure 15. The averaged FFT profile from all four orientations compared against a profile taken from the FEA simulation shown in Figure 13d). 
equivalent to the volume reduction; however, due to the increase in surface area of the optimised sample the full weight reduction is not gained.

Table 2. A summary and comparison table of the characteristics measured from the non-optimised (Arches) and optimised (Opt.) lightweighted mirrors

\begin{tabular}{|l|l|l|l|l|l|l|l|l|}
\hline \multirow{2}{*}{ Mirror } & \multicolumn{3}{|c|}{ Physical characteristics } & Form error $[\mathrm{nm}]$ & \multicolumn{3}{c|}{ Surface roughness Sq [nm] } \\
\cline { 2 - 9 } & Vol. $\left[\mathrm{mm}^{3}\right]$ & Area $\left[\mathrm{mm}^{2}\right]$ & Weight $^{a}[\mathrm{~g}]$ & PV & RMS & $2.5 \times$ Mag. & $5 \times$ Mag. & $50 \times$ Mag. \\
\hline \hline Arches & 3449 & 6014 & $9.2 / 14.3$ & 323 & 83 & 2.47 & 3.37 & 6.29 \\
\hline Opt. & 2347 & 6719 & $6.4 / 11.7$ & 204 & 31 & 5.22 & 6.04 & 9.63 \\
\hline
\end{tabular}

$a^{a}$ - weight before $\mathrm{NiP}$ coating/ weight after NiP coating

In regard to surface form error, the optimised design did produce the lower PV and RMS values; however, the equivalent surface roughness was not achieved on the optimised part and there is roughly a factor of 2 degradation when compared against the non-optimised part. The surface roughness was measured with the surface form error at ESRF using three magnifications $2.5 \times, 5 \times$ and $50 \times$ and is included in Table 2. During the polishing of the optimised mirror, a trade-off had to be made as to whether to prioritise surface roughness or surface form error and the latter was chosen, therefore the expected surface roughness from the NiP substrate was not achieved.

Upon completion of the optimised sample polishing, a trade off had to be made that in order to reduce the surface figure error the polishing time between each iteration had to be reduced which meant that the optimum surface roughness that is usually expected on NiP was not achieved.

The results obtained from this study highlight the potential of topology optimisation in the design of future mirror lightweighting; the ability to design a structure which is optimised for the forces applied to the part does have clear advantages. In the case of the optimised sample, a single uniform polishing pressure does not represent all the forces applied during the polishing process and therefore the simulation is probably too simplistic to gain the full advantage of this technique. However, TO is not the only innovative technique that can be applied to $\mathrm{AM}$ in lightweighting design and these are discussed below.

\section{FUTURE WORK: DESIGN OPTIMISATION}

This section presents three AM design routes that have potential towards mirror lightweighting.

1. Topology optimisation

2. Regular lattices/tessellating polyhedrons

3. Voronoi cells

\subsection{Topology optimisation}

The basic process of topology optimisation has been discussed in Section 3.2 and in Figure 9. Within this paper TO has been applied to minimise the total internal strain energy of the part for a given mass reduction and boundary conditions (applied force and fixtures); the model presented followed the guidelines provided by COMSOL Multiphysics. ${ }^{11}$ TO for mirror lightweighting is an established field with particular examples towards open back space-based mirrors described in Qu et al. $2016^{12}$ and Liu et al. 2015. ${ }^{13}$ A specific example which presents a similar work flow to this research is presented in $\mathrm{Hu}$ et al. $2017 ;^{14}$ here the lightweighting structure of a $2 \mathrm{~m}$ diameter sandwich mirror for space is described that is optimised to minimise the polishing print-through effect for a given set of fixtures. Future research in TO will endeavour to simulate a more accurate polishing, or diamond turning, environment with the intention to incorporate these communities in the design phase. 


\subsection{Regular lattices/ tessellating polyhedrons}

Perfectly tessellating polyhedrons are those that can be tessellated without incurring any voids (e.g. a cube) to create a lattice structure. Figure 16 provides two examples of perfectly tessellating polyhedrons that have been studied so far: the rhombic dodecahedron (Figure 16(a)) and the truncated octahedron 16(b). The selection of these polyhedrons for study is influenced by the fact that they represent structures which enclose the maximum volume for the minimum surface area - the perfectly tessellating equivalent of spheres. ${ }^{15}$
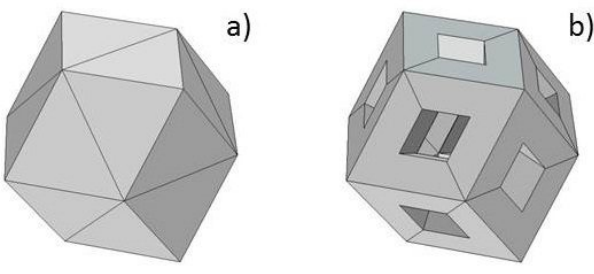

b)

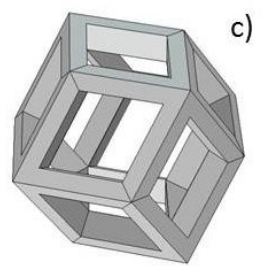

c)

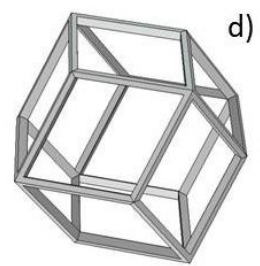

(a) Rhombic dodecahedron
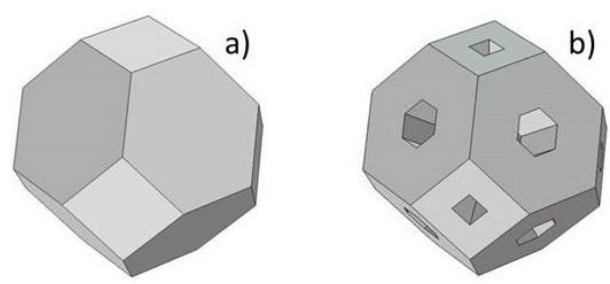
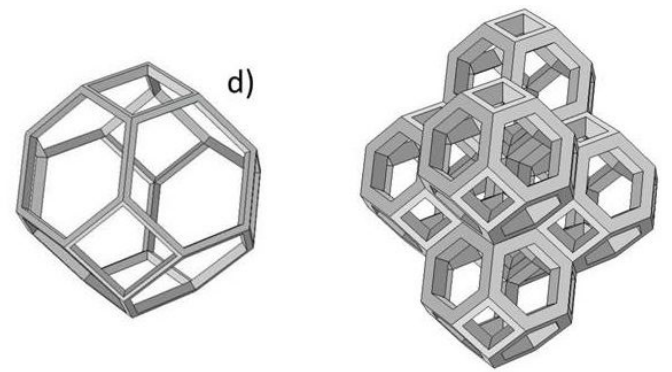

(b) Truncated octahedron

Figure 16. 16(a) and 16(b) (a) to $d)$ ) represent the density grading $(100 \%, 60 \%, 30 \%$ and $10 \%)$ that can be applied to the perfectly tessellating rhombic dodecahedron and truncated octahedron respectively. In both subplots the image on the far right demonstrates how the polyhedrons perfectly tessellate.

One advantage of dividing a lattice into individual polyhedrons is that it allows for density grading of a structure, as shown in Figure 16. Density grading would allow a lattice structure to be populated with polyhedrons with varying density based upon the strategic need for structural integrity in certain locations. For example, TO can be used to map where the critical regions are in a structure for a given load and this map can be used to distribute the different density polyhedrons. ${ }^{16}$ A worked example of this method, from simulation to printed part, when applied to a hammer handle can be found in COMSOL News (2015). ${ }^{17}$

\subsection{Voronoi cells}

The primary difference between the polyhedrons and Voronoi cells is that Voronoi cells offer an element of randomisation in the lightweighting structure. Voronoi cells are defined by a series of 'seeds' that have x, $\mathrm{y}$, and $\mathrm{z}$ coordinates. The cell is defined by a perimeter of straight lines, each straight line represents a perpendicular line located half way along a line which connects the seed of the cell with a neighbouring seed. If the seeds were distributed in a regular pattern, a Voronoi pattern would be a regular grid structure; however, by adding a random component to the seed placement a random Voronoi structure can be obtained. Voronoi cells can be considered as analogous to soap bubbles in a foam as demonstrated by Figure 17a).

Figure 17b) and $c$ ) highlight some early examples of how Voronoi cells have been implemented in lightweighting design. These early trials used MATLAB to create the Voronoi structures from a vector of seed coordinates calculated from the preferred spacing plus a random component. In Figure 17b) the cells have been density graded so that there are more smaller, densely packed cells near the mirror surface (top surface in the figure) 

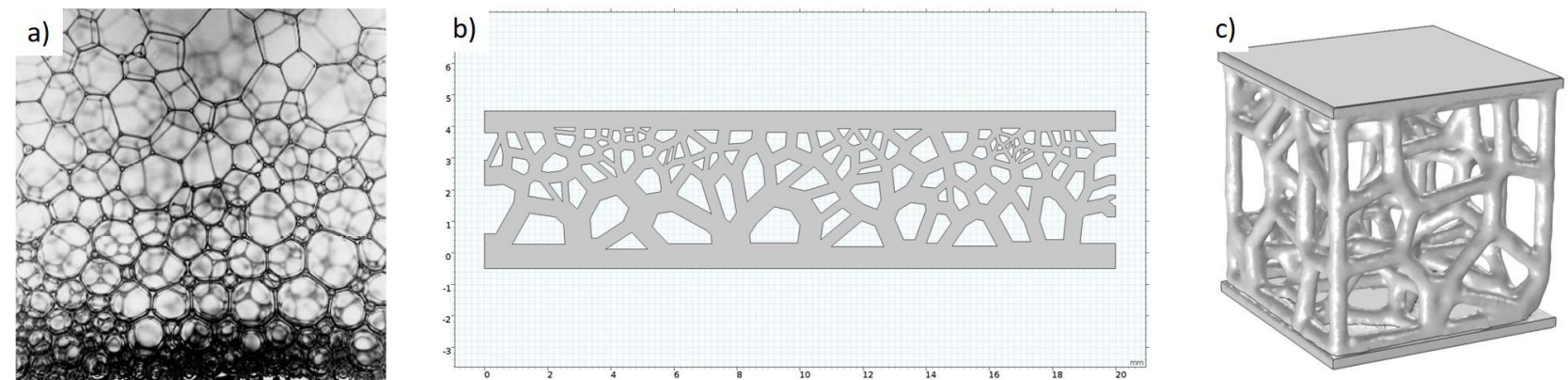

Figure 17. a) an example of 3 dimensional Voronoi cells in the form of soap bubbles (credit: $S S C$ ); b) a 2 dimensional representation of density graded Voronoi cells used as mirror lightweighting; and c) a cube composed of a $3 \times 3 \times 3$ matrix of Voronoi cells.

and fewer larger cells at the base. The density of the Voronoi cells can be graded via the same method as the polyhedrons using TO, or, as demonstrated in Mici, J. et al. (2015) ${ }^{18}$ a TO designed mirror mount, for example, can be further lightweighted by using Voronoi cells.

\subsection{Future work}

Three different lightweighting design routes have been presented and discussed. Future work will focus on converting these initial trials into proof-of-concept designs with the end goal that they will be printed as mirror lightweighting to provide design verification data.

\section{SUMMARY AND CONCLUSION}

The objective of this paper was to share experimental data and thoughts in the application of AM towards mirror lightweighting for space, to this end three themes were presented:

1. Diamond-turned mirror - this section discussed the surface metrology, particularly surface roughness, for an aluminium diamond-turned mirror and demonstrated the need to understand the orientation of the part within the build chamber in relation to surface roughness. The key result from this section was the $\sim 3.6 \mathrm{~nm}$ RMS roughness on the diamond-turned AM aluminium.

2. Lightweighting comparison: non-optimised versus optimised - in this section topology optimisation was introduced and how it can lead to a reduction in mirror weight while maintaining overall surface form. Metrology data evaluating surface form error was compared for a non-optimised and optimised sample pair. An analysis of the expected FEA print-through deformation in comparison to the measured deformation was described using Fourier space.

3. Future work: design optimisation - within this section three routes for further design optimisation were discussed as a means to ensure the optimum lightweighting design for an AM mirror is achieved. Each route represents research within the AM design community that will need to be adapted towards the mirror application.

Within this paper a series of mirrors have been presented which demonstrate that additive manufacturing can be used in mirror fabrication for visible and infrared wavebands in the future. However, only demonstrating reflectivity and low figure error does not showcase the full potential of AM mirrors; the advantage comes in the innovative structural design that can be implemented when the mirror design is no longer confined by traditional machining (mill, drill and lathe). Section 3 presented a first attempt in using topology optimisation to design a mirror lightweighting specifically to minimise the effects of polishing print-through. In Section 4 the breadth in lightweighting design potential was presented in terms of very early concept studies; these studies will be analysed and printed in the future with the goal of presenting alternative structures for mirror lightweighting. 
This paper has focused on using AM for mirror lightweighting; however, there is a much wider application of this technology in mirror design when the integration of additional functionality is considered. Future AM mirrors could demonstrate integrated optical mounting or embedded cooling channels; ultimately integrating functionality has the potential to minimise the number of fixtures and provide material continuity within a component and it is this innovation that is a long term goal of this research.

\section{ACKNOWLEDGMENTS}

C. Atkins would like to acknowledge the STFC Rutherford International Fellowship scheme which has provided the author the freedom to pursue this research. In addition, this project has received funding from the European Union's Horizon 2020 research and innovation programme under the Marie Skłodowska-Curie grant agreement No. 665593 awarded to the Science and Technology Facilities Council. M. Roulet's PhD position was funded under the H2020 program ERC-STG ICARUS 678777.

Finally the authors acknowledge the grant received from the UK Space Agency's National Space Technology Programme (NSTP3-PF-007) which has initiated this research.

\section{REFERENCES}

[1] Gibson, I., Rosen, D., and Stucker, B., [Additive manufacturing technologies], Springer, second ed. (2015).

[2] Sweeney, M., Acreman, M., Vettese, T., Myatt, R., and Thompson, M., "Application and testing of additive manufacturing for mirrors and precision structures," Proc. SPIE 9574, 957406-957406-13 (2015).

[3] Herzog, H., Segal, J., Smith, J., Bates, R., Calis, J., De La Torre, A., Kim, D. W., Mici, J., Mireles, J., Stubbs, D. M., and Wicker, R., "Optical fabrication of lightweighted 3d printed mirrors," Proc. SPIE 9573, 957308-957308-15 (2015).

[4] Hilpert, E., Hartung, J., Risse, S., Eberhardt, R., and Tünnermann, A., "Precision manufacturing of a lightweight mirror body made by selective laser melting," Precision Engineering 0141-6359 (2018).

[5] Vukobratovich, D., [Optomechanical Engineering Handbook - Chapter 5: Lightweight Mirror Design], CRC Press LLC (1999).

[6] Schwertz, K. and Burge, J. H., [Field Guide to Optomechanical Design and Analysis], SPIE Press (2012).

[7] Atkins, C., Feldman, C., Brooks, D., Watson, S., Cochrane, W., Roulet, M., Doel, P., Willingale, R., and Hugot, E., "Additive manufactured x-ray optics for astronomy," Proc.SPIE 10399, 10399 - 10399 - 15 (2017).

[8] Feldman, C. H., Atkins, C., Willingale, R., Brooks, D., Doel, P., Watson, S., and Cochrane, W., "Design and modeling of an additive manufactured thin shell for x-ray astronomy," in [Proc. SPIE], 10399(10399-53) (2017).

[9] Roulet, M., Atkins, C., Hugot, E., Lemared, S., Lombardo, S., and Ferrari, M., "3d printing for astronomical mirrors," Proc.SPIE 10675, 10675 - 10675 - 13 (2018).

[10] Friedman, E. and Miller, J. L., [Photonics Rules of Thumb], McGraw-Hill, second ed. (2004).

[11] COMSOL, M., "Topology optimization of an mbb beam." https://www.comsol.com/model/topologyoptimization-of-an-mbb-beam-7428 (2016).

[12] Qu, Y., Wang, W., Liu, B., and Li, X., "Topology optimization design of space rectangular mirror," Proc. SPIE 10154, 1015421-1015421-7 (2016).

[13] Liu, J. and Jiang, B., "Topology optimization design of a space mirror," Proc. SPIE 9795, 97952Y-97952Y$10(2015)$.

[14] Hu, R., Chen, W., Li, Q., Liu, S., Zhou, P., Dong, Z., and Kang, R., "Design optimization method for additive manufacturing of the primary mirror of a large-aperture space telescope," Journal of Aerospace Engineering 30(3), 04016093 (2017).

[15] Pearce, P., [Structure in nature is a strategy for design], The MIT Press, fifth ed. (1990).

[16] Panesar, A., Abdi, M., Hickman, D., and Ashcroft, I., "Strategies for functionally graded lattice structures derived using topology optimisation for additive manufacturing," Additive Manufacturing 19, 81-94 (2018).

[17] Carver, L. and COSMOL, "Virtual material design in 3d printing makes headway with multiscale modeling." COMSOL News (2015). 
[18] Mici, J., Rothenberg, B., Brisson, E., Wicks, S., and Stubbs, D. M., "Optomechanical performance of 3dprinted mirrors with embedded cooling channels and substructures," Proc.SPIE 9573, 9573 - 9573 - 14 (2015).

Proc. of SPIE Vol. 10706 107060I-17

Downloaded From: https://www.spiedigitallibrary.org/conference-proceedings-of-spie on 7/12/2018 Terms of Use: https://www.spiedigitallibrary.org/terms-of-use 